\title{
VARIA
}

\section{APRENDIZ DE FRESCOS. NOTICIA SOBRE LA PRESENCIA DE GASPAR BECERRA EN LA ESTUFA DE LA ALHAMBRA}

\author{
NuRIA MARTÍNEZ JiMÉNEZ ${ }^{1}$ \\ Universidad de Granada
}

En este artículo damos a conocer la noticia sobre la presencia de Gaspar Becerra en Granada constatando su acer-
camiento a la pintura mural italiana antes de su marcha a Italia.
Palabras clave: Gaspar Becerra; Julio Aquiles; Alexander Mayner; Estufa de la Alhambra; Granada; pintura mural.

\section{MURAL PAINTING APPRENTICE: NEWS CONCERNING THE PRESENCE OF GASPAR BECERRA IN THE ESTUFA OF THE ALHAMBRA}

This article presents the first news about the presence of Gaspar Becerra in Granada, prior to his move to Italy. In this way the author verifies that Becerra already knew Italian mural painting in Spain.

Key words: Gaspar Becerra; Julio Aquiles, Alexander Mayner; Alhambra; Granada; mural painting.

Como citar este artículo / Citation: Martínez Jiménez, Nuria (2018): “Aprendiz de frescos. Noticia sobre la presencia de Gaspar Becerra en la Estufa de la Alhambra". En: Archivo Español de Arte, vol. 91, núm. 361, Madrid, pp. 65-69. https://doi.org/10.3989/aearte.2018.05

Gaspar Becerra, es uno de los artistas más importantes e influyentes del Renacimiento español. Desde su muerte, los escritos de numerosos teóricos han dejado constancia del excepcional legado del artista. Entre ellos destacan Giorgio Vasari (con quien colaboró en la ornamentación del Palacio de la Cancillería de Roma y cuya referencia documental es básica para el conocimiento de sus trabajos en Italia), Juan de Arfe, Lázaro Díaz del Valle, Pablo Céspedes, Francisco Pacheco ${ }^{2}$, Antonio Palomino ${ }^{3}$ Juan Agustín Ceán Bermúdez ${ }^{4}$, entre otros eruditos.

A principios del siglo XX la biografía publicada por Elías Tormo ${ }^{5}$ abrió la puerta a nuevas investigaciones sobre el artista, centradas, principalmente, en la repercusión que la formación

1 nuriamj@correo.ugr.es /ORCID iD: http:orcid.org/0000-0003-2479-7598. Trabajo realizado en el marco del proyecto I+D HAR2014-52061-PD "La copia pictórica en la Monarquía Hispánica (siglos XVI-XVIII) dirigido por el doctor David García Cueto y financiado por el Ministerio de Economía y Competitividad.

2 Pacheco, 1990: 349.

3 Palomino, 1988: 46-53.

4 Ceán, 1800: pp. 107-116.

5 Tormo, 1912: 241-265. González, 1969: 327. 
adquirida en Italia tuvo para la introducción del romanismo en España, ensalzando el excepcional retablo de la Catedral de Astorga ${ }^{6}$. Con todo, en la última década del siglo, comenzaron a realizarse artículos y monografías profundizando en su faceta como muralista en Italia ${ }^{7}$ y, más tarde, en el palacio del Pardo en Madrid ${ }^{8}$.

A la vista de este amplio panorama historiográfico, no cabe duda que se ha avanzado considerablemente en el conocimiento de la actividad pictórica de Becerra -ámbito artístico que nos interesa principalmente-, pero lo cierto es que todavía queda bastante por decir sobre ella y, precisamente, dentro de ese contexto, este trabajo pretende aportar datos inéditos sobre su formación antes de marchar a Italia.

No hay constancia de que Gaspar Becerra realizara ningún trabajo en España antes de llegar a Roma. En efecto, las investigaciones realizadas sobre su entorno familiar ${ }^{9}$ muestran que, su padre Antón Becerra, pintor de Baeza, no pudo enseñar a sus hijos las innovaciones pictóricas que comenzaban a inundar los talleres de Sevilla ${ }^{10}$ o Granada. Por consiguiente, tradicionalmente se ha abogado por que Becerra se formara en Italia. Concretamente, Elías Tormo planteó que Becerra viajó a Italia, en torno a 1540, para estudiar con Miguel Ánge ${ }^{11}$ y de ahí pasar al taller de Vasari12 ${ }^{12}$, puesto que en España hubiera sido imposible aprender la pintura al fresco ${ }^{13}$.

Desde la primera década del siglo XXI, se afirma la tesis de la inexistencia de un vínculo directo entre Miguel Ángel y Becerra, a la vez que se está contribuyendo a documentar la estancia italiana. Con todo, su formación en España ha continuado siendo una incógnita solventada a través de sugestivas hipótesis que intentan desvelar las fuentes renacentistas de las que Becerra pudo beber antes de su marcha ${ }^{14}$.

En este contexto, la noticia que hemos hallado en el Archivo de la Alhambra ${ }^{15}$ revela que Gaspar Becerra no sólo conocía las obras de Julio Aquiles y Alexander Mayner, sino que recibió su formación temprana como muralista a la sombra de los artistas italianos que trabajaron en la Alhambra.

Julio Aquiles y Alexander Mayner habían llegado a España en $1533^{16}$ para trabajar en las casas de Francisco de los Cobos en Valladolid y Ubeda. Sin embargo, la relevancia de las obras

\footnotetext{
${ }^{6}$ Fracchia, 1998: 132-152. García, 1999: 177-206. Arias, 2015: 423-440.

7 Redín, 2002: 129-144; 2007; 2010: 235-248. Salort, 2005: 100-102.

8 González, 1962: 1-20. López. 1991. Martínez, 1991: 197. Checa, 2005. Rodríguez, 2005: 54-73. Fernández-Toribio, 2004: 739-746.

9 Herrera, 2004: 59-96. Viribay, 2003: 585-625.

10 Resulta curioso advertir que Antón Becerra envió a su hijo Juan a estudiar a Sevilla junto con el pintor cordobés Pedro Fernández mientras que, a la luz de la documentación actual, Pedro y Gaspar Becerra quedaron en el taller de Antón en Baeza. Angulo, 1944: 243.

11 Uno de los primeros en citar esta relación es Pablo Céspedes cuando indica "que fue un gran imitador de Miguel Ángel en pincel y en escultura". Redín, 2007: 159. Años más tarde, Francisco Pacheco escribe que superó a Berruguete "por haber seguido a Miguel Ángel". Pacheco, 1990: 345. Una vinculación que también recogió Antonio Palomino al mencionar que en su marcha a Italia estudió las obras de Miguel Ángel, "de quien fue discípulo, aunque también de Rafael de Urbino". Palomino, 1988: 46.

12 Tormo, 1912: 126.

13 Tormo, 1912: 122.

14 Gonzalo Redín propone varias hipótesis para buscar las raíces de la formación del artista baezano. Por una parte plantea que fuera el propio Pedro Machuca quien propusiera a Antón Becerra la estancia de Gaspar en Italia; además sugiere que Gaspar Becerra conociera las pinturas de Julio y Alexander. Redín, 2007: 160-161. Por consiguiente la noticia aportada corrobora la conexión entre dichas hipótesis.

15 Manuel Gómez Moreno fue el primero en consultar las nóminas, tal y como se evidencia en las publicaciones del Boletín del Centro Artístico de Granada. En ellas documentó las fechas, la presencia de los pintores italianos y citó la relación de algunos materiales. Sin embargo, en las investigaciones que estamos realizando en hemos profundizado en el análisis de la documentación, la utilización de los materiales, así como en la presencia de colabores tan destacados como el protagonista de este artículo.

${ }^{16}$ La primera referencia se encuentra a mediados de 1533 cuando Alonso Berruguete elige a Julio Aquiles para tasar el retablo de San Benito de Valladolid. Gómez, 1887: 190.
} 
en las estancias del Emperador, dirigidas por Luis Hurtado de Mendoza, III Conde de Tendilla, los condujo a Granada tras concluir las pinturas de Valladolid.

La presencia de Aquiles y Mayner en Granada está documentada en $1537^{17}$, pero su llegada debió producirse meses antes. Rosa López Torrijos propuso que las pinturas del cuarto de las frutas "debieron ser realizadas en torno a 1535-1537, por uno o ambos pintores llegados de Italia"18. En efecto, estas salas fueron las primeras en ser decoradas, puesto que en agosto de 1537 se dice que las "cuadras que ya están doradas"19.

El proceso de elaboración de las pinturas, también puede seguirse a través de los cuadernos de nóminas. Por ello, podemos concretar que el primer espacio pintado fueron las cuadras nuevas, concluidas en octubre de 1537. Después, continuaron los trabajos de ornamentación de los corredores de las cuadras (que terminaron de dorarse en agosto de 1539) y del corredor de la Estufa (iniciado en octubre de 1537 y concluido en 1539, aunque Alexander Mayner lo retocó en abril de 1541). Los últimos trabajos se centraron en la pintura de la Estufa, iniciada en diciembre de 1539, que supuso la culminación de los trabajos de los pintores en julio de $1544^{20}$.

Un año especialmente relevante en el proceso de ejecución de las obras es 1540, pues hemos detectado que tras la ausencia de los pintores en la Alhambra, durante el mes de abril de ese año ${ }^{21}$, se produjo un cambio de dirección.

Tradicionalmente se identifican los trabajos de Aquiles en las cuadras y de los corredores por sus características estéticas, pero además en las nóminas se advierte como Julio deja de aparecer en primer lugar, reduciendo además sus jornadas para dar paso al protagonismo de Alexander. Esto sugiere que los pintores habían diversificado su trabajo. Como mencionamos anteriormente, Francisco de los Cobos había contratado a los artistas para ornamentar su palacio en Úbeda. Aunque, no hay restos materiales o documentales de las pinturas del palacio ubetense, el propio Palomino rubrica que: "ellos mismos pintaron las casa de los Cobos, en la ciudad de Úbeda"22. Estos murales debieron realizarse con anterioridad a $1545^{23}$. Por consiguiente, la ausencia de los pintores en abril de 1540, unido a la reducción de jornadas de Julio en Granada y a la llegada de colaboradores procedentes de la Lona — como mostraremos a continuación-, sugiere que los pintores comenzaran a trabajar en Úbeda y Granada de forma simultánea. Julio se centraría en la ejecución de las pinturas del Palacio de Cobos en Úbeda y Alexander concluiría las de Granada. De esta forma, no se interrumpió la colaboración de Aquiles en Granada explicando, además, porqué en agosto de 1541 ambos están ausentes de la Estufa, este paréntesis pudo coincidir con una revisión de las obras en Úbeda.

Retomando la actividad en la Alhambra a partir de 1540, también detectamos un hecho verdaderamente relevante. Los pintores granadinos y foráneos consolidados como colaboradores en la etapa anterior: Pedro Robles, Miguel Quintana y Nicolás de Génova, reducen sus intervenciones en la Alhambra dando paso a colaboradores puntuales como: Juan Paez ${ }^{24}$, Pedro Sánchez ${ }^{25}$ y, lo más llamativo, a los hermanos Becerra, Pedro y Gaspar.

17 Año en el que empiezan a registrase la primeras nóminas de los trabajadores de la Alhambra.

18 López, 1986: 42.

19 Nóminas de agosto 1537, Archivo del Patronato de la Alhambra y el Generalife (APAG),Capitanía General del Reino de Granada y Alcaldía de la Alhambra, caja 1, legajo 22, fol. 9v.

${ }^{20}$ Nóminas de julio 1544, APAG, Capitanía General del Reino de Granada y Alcaldía de la Alhambra, caja 2, legajo 13 , fol. $27 \mathrm{v}$.

21 "Los pintores no se echan este mes porque no trabajaron en él". Nóminas de abril 1540, APAG, Capitanía General del Reino de Granada y Alcaldía de la Alhambra, caja 1, legajo 40, fol. 23v.

22 Palomino, 1988: 35.

23 Año en el que Julio Aquiles fue contratado para trabajar en la Capilla del Camarero Vago y cuya residencia en Úbeda está contrastada. Para conocer más sobre el extraordinario impulso que tuvo la pintura mural en Úbeda véase. Mendoza, 2002: 83-109. Almansa, 2008.

${ }^{24}$ Nóminas de diciembre 1540, APAG, Capitanía General del Reino de Granada y Alcaldía de la Alhambra, caja 1, legajo 45, fol. 20.

${ }_{25}$ Pedro Sánchez es el único colaborador que aparece en varias ocasiones. Nóminas de enero de 1542, APAG, Capitanía General del Reino de Granada y Alcaldía de la Alhambra, caja 2, legajo 5, fol. 23v. y en Nóminas de marzo 
En el caso de los Becerra, su incursión en el círculo de Aquiles y Mayner pudo venir respaldada por Pedro Machuca, maestro de obras de la Alhambra, quien gozaba de una buena relación personal con Antón Becerra ${ }^{26}$. En efecto, mientras Antón Becerra trabajaba en el retablo de la iglesia de la Vera Cruz de Baeza ${ }^{27}$ representó a Machuca en la tasación del retablo de Nuestra Señora de la Capilla de la iglesia parroquial de San Ildefonso de en $1540^{28}$.

Probablemente, la cercanía de Baeza y Úbeda, facilitó el acceso de jóvenes pintores al círculo de Aquiles, con quien aprendieron la técnica del fresco en la casa de los Cobos de Úbe$\mathrm{da}^{29}$ acompañándolo, además, en sus viajes a Granada para pintar en la Estufa. Entre estos colaboradores hemos documentado, en junio de $1542^{30}$, la actividad de Gaspar Becerra moliendo revoco en la Estufa de la Alhambra, junto a Diego de Villanueva. Pedro Becerra ${ }^{31}$ participará en noviembre del mismo año, coincidiendo con la última nómina recibida por Julio Aquiles en la Alhambra ${ }^{32}$.

De lo expuesto se deduce que la estancia en Granada de Gaspar Becerra fue muy puntual, por lo que en la Alhambra no pudo adquirir una experiencia en profundidad. Sin embargo, no cabe duda de que la formación adquirida en la cercanía de los maestros italianos (tanto en Granada como en Úbeda), le brindó la posibilidad de conocer in situ el proceso de realización de las pinturas murales al fresco y de aprender la esencia de ésta técnica. Cualidades que, años más tarde, con mayor madurez, le posibilitaron equipararse a los pintores de la Cofradía de San Lucas de Roma e integrarse en los círculos artísticos y humanistas de la capital italiana.

\section{BIBLIOGRAFÍA}

Almansa Moreno, José Manuel (2008): Pintura mural del Renacimiento en el Reino de Jaén. Jaén: Instituto de Estudios Giennenses.

Angulo Iñiguez, Diego (1944): "Pintores cordobeses del Renacimiento". En: Archivo Español de Arte, 17, 64. Madrid, pp. 226-244.

Arias Martínez, Manuel (2007): "Miscelánea sobre Gaspar Becerra”. En: Boletín del Museo Nacional de Escultura, 11, pp. 7-15.

Arias Martínez, Manuel (2015): "Los modelos tridimensionales de Gaspar Becerra y la uniformidad del Romanismo en España”. En: Hispanic Research Journal, 16, 5, Terence O’ Reilly, pp. 423-440.

Ceán Bermúdez, Juan Agustín (1800): Diccionario de los más ilustres profesores de las Bellas Artes en España. Tomo 1. Madrid: Imprenta de la Viuda Ibarra.

Checa, Carmen García-Frías (2005): Gaspar Becerra y las pinturas de la Torre de la Reina del Palacio de El Pardo: una nueva lectura tras su restauración. Madrid: Patrimonio Nacional.

Fracchia, Carmen (1998): "La herencia italiana de Gaspar Becerra en el retablo mayor de la Catedral de Astorga". En: Anuario del Departamento de Historia y Teoría del Arte, 9-10, Madrid, pp. 132-152.

García Gainza, María Concepción (1999): "El retablo de Astorga y la difusión del romanismo". En: Boletín del Museo e Instituto Camón Aznar, 78-79, Zaragoza, pp. 177-206.

Gómez Moreno, Manuel (1887): "Los pintores Julio y Alejandro y sus obras en la casa Real de la Alhambra” (1). En: Boletín del Centro Artístico de Granada, 2, 22, pp. 189-195.

1540, APAG, Capitanía General del Reino de Granada y Alcaldía de la Alhambra, caja 2, legajo 6, fol. 21v.

${ }^{26}$ Gonzalo Redín plantea que Machuca le recomendara el viaje a Italia, pero pensamos que es más posible que primero le recomendara el conocimiento de las obras de Granada y, tras demostrar sus habilidades y pericia, la marcha a Italia. Redín, 2007: 161.

27 Realizado entre 1531-1541. Actualmente se piensa que las tablas de Antón Becerra conservadas en el Museo Catedralicio de Baeza pertenecerían a este retablo. Sánchez, 2003: 242-243; 256-257.

28 Tasación retablo. La relación de Antón y la catedral de Jaén se remonta a 1511. Lázaro, 2009: 296.

29 Redín, 2007: 161.

${ }^{30}$ Nóminas de marzo 1540, APAG, Capitanía General del Reino de Granada y Alcaldía de la Alhambra, caja 2, legajo 7 , fol. $15 \mathrm{v}$.

31 Nóminas de marzo 1540, APAG, Capitanía General del Reino de Granada y Alcaldía de la Alhambra, caja 2, legajo 10. fol. $3 \mathrm{v}$.

32 El año 1543 no está en el archivo y en 1544 sólo trabaja Alexander. 
González Martín., Juan José (1962): "El Alcázar de Madrid en el siglo XVI”. En: Archivo Español de Arte, 35, 137, pp. 1-20.

González Martín, Juan José (1969): "Precisiones sobre Gaspar Becerra”. En: Archivo Español de Arte, 42, 168, Madrid, pp. 327-356.

Herrera García, Francisco Javier (2004): "Gaspar Becerra: su entorno familiar y profesional a la luz de nuevas fuentes documentales". En: Boletín del Museo e Instituto "Camón Aznar", 93, Zaragoza, pp. 59-96.

Huecas Fernández-Toribio, M. ${ }^{a}$ Eugenia (2006): "Descubrimiento de pinturas murales del siglo XVI en el Torreón de Gaspar Becerra del Palacio Real del Pardo de Madrid”. En: Arqueología, arte y restauración: actas del IV Congreso Internacional "Restaurar la Memoria", Valladolid, pp. 739-746.

Lázaro Damas, María Soledad (2009): "La obra documentada de Pedro Machuca y Luis Machuca Horozco en la Catedral de Jaén (1539-1550)” En: Boletín. Instituto de Estudios Giennenses, 198, Jaén, pp. 289-319.

López Torrijos, Rosa (1986): "La escuela de Rafael y el bodegón español”. En: Archivo Español de Arte, 59, 233, pp. 32-52.

López Torrijos, Rosa (1991): Las pinturas de Becerra en el Palacio del Pardo y la Iconografía de Perseo y Pegaso. Madrid: CSIC. Alpuerto.

Martínez Martínez, Araceli (1991): "Un fresco de El Pardo atribuido a Vicente Carducho, documentado como de Luis de Carvajal”. En: Archivo Español de Arte, 64, 254, Madrid. p. 197-201.

Mendoza, Arsenio (2002): "La pintura mural en la ciudad de Úbeda en el siglo XVI: una aproximación histórica”. En: Laboratorio de Arte, 15, Sevilla, pp. 83-109.

Pacheco, Francisco (1990): El arte de la pintura. Madrid: Cátedra.

Palomino, Antonio (1988): Museo pictórico y escala óptica. El parnaso español laureado. Madrid: Aguilar.

Redín Michaus, Gonzalo (2002): "Sobre Gaspar Becerra en Roma. La capilla de Constantino del Castillo en la iglesia de Santiago de los Españoles”. En: Archivo Español de Arte, 75, 298, Madrid, pp. 129-144.

Redín Michaus, Gonzalo (2007): Pedro Rubiales, Gaspar Becerra y los pintores españoles en Roma, 1527-1600. Madrid: Editorial CSIC.

Redín Michaus, Gonzalo (2010): "El testamento y otros documentos sobre Daniele da Volterra". En: Archivo Español de Arte, 83, 331, Madrid, pp. 235-248.

Rodríguez Arana Esperanza (2005): "Recuperación y restauración de la decoración del Torreón de la Reina en el Palacio Real de El Pardo". En: Reales Sitios: Revista del Patrimonio Nacional, 2005, 165, Madrid, pp. 54-73.

Salort Pons, Salvador (2005): “Gaspar Becerra en Florencia”. En: Archivo Español de Arte, 78, 309, pp. 100-102.

Sánchez Concha, Francisco José (2003): Pinturas de caballete de la catedral de Baeza. Baeza: Ayuntamiento de Baeza.

Tormo, Elías (1912): "Gaspar Becerra. Notas varias". En: Boletín de la Sociedad Española de Excursiones, 21, 4, pp. 241-265.

Viribay, Miguel (2003): “Aproximación a Gaspar Becerra, romanista de Baeza (1520-1568)”. En: Boletín del Instituto de Estudios Giennenses, 168, pp. 585-625.

Fecha de recepción: 20-X-2016

Fecha de aceptación: 17-I-2017 